\title{
Riflessi di Cosimo Fanzago a Salamanca*
}

\author{
Marina Bozzi Corso \\ Università del Salento
}

RESUMEN. El presente artículo versa sobre la presencia de los trabajos de Cósimo Fanzago en España, en particular sobre la obra virreinal del presbiterio de la iglesia de las Agustinas de Salamanca. Tras encuadrar el tema, se formula una hipótesis sobre la forma original del cancel mayor y los trabajos del presbiterio con la intención de hacer su reconstrucción virtual. Al final del artículo se analiza la influencia del modelo fanzagiano utilizando como herramienta de lectura la recepción de los modelos del Barroco napolitano en el arte castellano.

Palabras clave: Obra en metal, Cósimo Fanzago en Salamanca, influencias.

ABSTRACT. The paper deals with Cosimo Fanzago's opera, particularly with the important vice-regal order regarding the presbytery of the church Las Agustinas in Salamanca. After an historical-artistic background, I will discuss an hypothesis about the original shape of the big disappeared gate and about the other works of the presbytery, aiming to rebuild them virtually. I will then analyse the fortune of Fanzago's model in Castilian art by interpreting how in loco Neapolitan Barocco was perceived.

Key words: Cosimo Fanzago's metal works in Salamanca, fortune.

RÉSUMÉ. In questo articolo intendo parlare della presenza di opere di Cosimo Fanzago in Spagna, in particolare dell'importante commissione vicereale relativa al presbiterio della chiesa de las Agustinas di Salamanca. Dopo un inquadramento della questione, cerchero' di formulare un'ipotesi sulla forma originaria della grande cancellata scomparsa e delle altre opere del presbiterio, in previsione di un tentativo di ricostruzione virtuale. Infine cerchero' di analizzare la fortuna del modello fanzaghiano nell'arte castigliana attraverso una possibile chiave di lettura di come venissero recepiti in loco i modelli del barocco napoletano..

Parole chiave: Opere in metallo, Cosimo Fanzago a Salamanca, riflessi.

In una lettera indirizzata ad Ulisse Prota Giurleo del 30 Novembre 1957 Rober-

\footnotetext{
* Questo contributo è un primo e parziale risultato dei mei studi e ricerche svolte in Spagna grazie ad una borsa di studio del Programma Socrates/Erasmus 2005/2006 alla quale ho concorso dietro suggerimento e incoraggiamento di Regina Poso. Voglio ringraziare con tutto il cuore i colleghi e gli amici del Dpto. de Patrimonio Artístico y Documental dell'Università di Leon che mi hanno sempre dimostrato stima, disponibilità e affetto, soprattutto nei momenti difficili, in un periodo molto bello della mia vita, di cui ho già tanta nostalgia.
}

to Longhi scriveva da Firenze : "Illustre e caro Professore, I documenti da Lei trovati per l'opera del Fanzago a Salamanca sono davvero importantissimi e sarà un piacere averli presto a stampa col suo dotto commento. Ho dunque studiato attentamente le fotografie inviatemi e che ora Le rimando. Nessun dubbio che la Purisima del Ribera è firmata e datata 1635, come risulta da un particolare fotografico pubbl. a fig. 66 della monografia sul Ribera della Gué Trapier (New York,1952). Quanto al resto del retablo ecco quanto posso dirle con l'ausilio di una lente : La Pietà nella cimasa è anch'essa di 
invenzione riberiana, ma sembra soltanto della sua scuola. Sui lati : in alto a sinistra : Incontro di Gioacchino e Anna, Napoletano o spagnuolo, ma la riprod. Non consente di dir altro. In alto a destra: San Giov. Battista in piedi in un paesaggio. E' una composizione certamente di origine reniana; la qualità ne sembra buona e non è impossibile si tratti di un originale del Reni stesso nel suo periodo avanzato; certo dopo il '30. In basso a sinistra: San Giuseppe col Bambino. Sembra quadro italiano, ma non direi napoletano. Le maggiori somiglianze sono con Alessandro Turchi detto L'Orbetto (Veronese). In basso a destra : Sant'Agostino col fanciullo sulla riva del mare. Questo invece risulta essere dipinto di cerchia del Rubens. Una composizione del Rubens simile a questa, ma invertita nella composizione, e datata del 1637, è al Rudolphinum di Praga, e Lei la può vedere a fig. 422 del Rubens della serie $<<$ Massiker der Kunst $>$. Da queste osservazioni risulta che il retablo, come spesso avveniva in Ispagna, fu composto a guisa di piccola pinacoteca di autori diversi. Ci si serviva spesso a questo fine di quadri importanti dall'Italia o dalla Fiandra, e il Monterey ne aveva una buona raccolta" ${ }^{1}$. In realtà questa lettera fu pubblicata dallo stesso Prota Giurleo di lì a poco in margine ad un suo articolo in cui si veniva a conoscenza per la prima volta dei documenti relativi ad alcune opere di Cosimo Fanzago (Clusone 1591-Napoli 1678), fino ad allora ignorate ed escluse dal catalogo dell'artista bergamasco, commissionategli nel 1633 dal Conde de Monterrey, viceré di Napoli dal 1631 al 1637, per Salamanca ${ }^{2}$. Tra queste

\footnotetext{
${ }^{1}$ U. Prota GIURleo, "Fanzago ignorato", in Il Fuidoro, 4, 1957, pp.146-150, p.149.

${ }^{2}$ Cfr. Idem. Si tratta di alcuni documenti della sezione Notai Antichi dell'Archivio di Stato di Napoli ed in particolare di quelli del fondo del notaio Andrea Fasano riediti in U. PROTA GIURLEO, "Cosimo Fanzago", Ricerche sul '600 Napoletano, 1986, pp. 9-31. L'importanza di questi documenti verrà poi evidenziata in un articolo di Angela Madruga Real (A. MADRUGA REAL, "Cosimo Fanzago en las Agustinas de Salamanca", Goya, 125, 1975, pp. 291-297 ), che è anche l'autrice della bella monografia sul complesso della
}

brillava di luce propria il magnifico retablo concepito dal Fanzago per una cappella nella Chiesa de las Ursulas e che sarebbe stato poi collocato, invece, sull'altare maggiore della Chiesa de las Agustinas Descalzas, con al centro, como una epifanía deslubrante ${ }^{3}$, l'imponente e bellissima Immacolata del Ribera, circondata dagli altri dipinti di formato più piccolo (Lám. 1). Lo studioso napoletano che aveva richiesto al Longhi un parere sulla paternità delle tele, di cui possedeva solo immagini deducibili da una riproduzione fotografica dell'intero retablo, ebbe comunque da questa autorevole ma affettuosa risposta la certezza che si trattasse di dipinti di scuola fiamminga e italiana,

chiesa e convento de las Agustinas di Salamanca (A. MADRUGA REAL, Arquitectura barroca salmantina. Las Agustinas de Monterrey, Salamanca, 1983). Per lo studio di questo argomento si sono, inoltre, rivelati molto utili i seguenti studi : P. FOGACCIA, Cosimo Fanzago, Bergamo, 1945; A. GARCÍA BOIZA, Una Fundacion de Monterrey. La iglesia y convento de M.M. Augustinas de Salamanca, Salamanca, 1945; M. GÓMEZ MORENO, Catalogo monumental de Espana. Provincia de Salamanca, Madrid, 1967; R. WITTKOWER, Arte e architettura in Italia. 1600-1750, Torino, 1972; G. CANTONE, Napoli Barocca e Cosimo Fanzago, Napoli, 1984; D. DomBrowsKI, “Napoles en España. Cosimo Fanzago, Giuliano Finelli, las esculturas del Altar Mayor en las Augustinas Descalzas de Salamanca y un monumento funerario desaparecido", Anuario del Departamento de Historia y Teoria del Arte (U.A.M.), VII-VIII, 1995-1996, pp.87-93; A. NAVA CellinI, "La scultura dal 1610 al 1656", La scultura del Seicento a Napoli, Torino, 1997, pp.15-55; A.SPINOSA, "Cosimo Fanzago, Lombardo a Napoli", Idem, pp. 57-82; E. CATELLO, "Cosimo Fanzago : puntualizzazioni e ipotesi su alcuni problemi di scultura", Ricerche sul '600 napoletano, 2002(2003), pp.21-28; P. D'AGOSTINO, "Un bronzetto spagnolo di Cosimo Fanzago a New York", Prospettiva, 109, 2003(2004); I. MiguÉLIZ VALCARLOS, "Bustos relicarios italianos en el Museo Lázaro Galdiano", Goya, 310, 2006, pp. 3-10.

${ }^{3}$ Così ebbe a dire Antonio García Boiza commentando la tela della Purísima del Ribera, firmata e datata "JUSEPE DE RIBERA, ESPAÑOL VALENCIANO. F. 1635." , considerata dalla critica "como la mas portentosa obra de la pintura barroca mariana del mundo" (A. GARCíA BOIZA, cit., p. 26). Sui dipinti e relativa bibliografia si veda : A. MADRUGA REAL, Arquitectura barroca salmantina., cit., pp.155-162; P. D’AGOSTINO, cit., pp. 83-84. 
non tutti napoletani ${ }^{4}$. Sebbene fossero ancora gli anni Cinquanta del Novecento e gli studi sulla chiesa della Purísima Concepción di Salamanca ancora agli esordi, le parole del Longhi confermavano il noto mecenatismo di Don Manuel de Zuñiga y Fonseca, sesto conte di Monterrey, che in questa sede è l'aspetto che più mi interessa. Appassionato d'arte, soprattutto di scultura e di pittura italiana, ma non solo, come vedremo, il viceré, dalla personalità complessa ma interessante, Principe a dire il vero che aveva bassa statura e pensieri giganti, riuscì ad intrattenere, soprattutto a Napoli, rapporti stretti e significativi con gli artisti più importanti allora attivi nel Regno ${ }^{5}$.

${ }^{4}$ Nella lettera citata il Longhi esprime un parere anche sugli altri dipinti della chiesa : "Quanto agli altri altari : "Il San Gennaro del Ribera venne dal Mále riferito al 1631, perché rappresenta l'eruzione del Vesuvio; ma ciò non è stringente e il dipinto può essere benissimo posteriore anche al 1635. L'Annunciazione è certamente del Lanfranco, e anche il Voss la dice fatta a Napoli, dove l'artista giunse nel 1634. Come Lei sa inoltre, dalle lettere del Lanfranco a Ferrante Carlo (pubbl. nella racolta del BottariTicozzi) risulta ben chiaro che il Monterey apprezzava e proteggeva molto il Lanfranco. Dalle stesse lettere risulta anche la rottura avvenuta tra Il Lanfranco e il Fanzago per via del matrimonio della figlia del Lanfranco con Giuliano Finelli, mentre il Fanzago desiderava darla a suo figlio. Tutto questo sembra essere avvenuto proprio mentre si lavorava per il Monterey."(Cfr. U. PROTA GIURLEO, "Fanzago ignorato", cit., p.149).

${ }^{5} \mathrm{La}$ frase che ho riportato in corsivo è del Parrino ed è citata in U. PROTA-GIURLEO, "Fanzago ignorato", cit., p.146. Sulla figura del Monterrey cfr. : A. MADRUGA REAL, "Cosimo Fanzago", cit.; A. MADRUGA REAL, Arquitectura barroca salmantina., cit. Esponente dell'alta nobiltà castigliana, cognato di Don Gaspar de Guzman, Conde-Duque d'Olivares e Ministro di Filippo IV, di cui sposerà la sorella doña Leonor María de Guzman y Pimentel. Farà il suo primo viaggio in Italia, che coincide con il suo primo soggiorno romano di nove mesi, nel 1622, come ambasciatore della Corona nello Stato Vaticano, carica diplomatica che manterrà fino al 1631, mentre svolgerà la sua carriera militare nella frontiera di Estremadura e quella politica a Napoli dove giunge alla fine dell'aprile del 1631. Valoroso combattente, amante de la vida en toda sus facetas, il Conde è insieme timoroso della malattia e della morte come si evince dalla sua corrispondenza con le Agustinas, ma come emerge pure dal profilo di Gonzáles-
Ma dal 1957 alcune cose sono cambiate negli studi su questo argomento; allo stato attuale delle ricerche la tradizione critica conta più di un contributo notevole, che ha ampliato e per qualche aspetto modificato le conoscenze sul Monterrey e l'opera salmantina, un conjunto artistico che la Madruga Real, come già ricordava il Dombrowski, ha definito "un puente artistico entre Napoles y Salamanca" ${ }^{\prime 6}$. Ma se gli studi di architettura, pittura e scultura sul complesso della chiesa e del convento de las Agustinas Descalzas, ora Agustinas Recoletas, sono andati molto avanti, un settore ancora poco indagato resta quello delle opere in metallo. Le opere realizzate dagli artisti e dalle maestranze italiane per l'arredo sacro in metallo del presbiterio della chiesa sono gia' state rese note in Italia da Alvar Gonzáles-Palacios, Paola Giusti, Paola D'Agostino ${ }^{7}$; non sempre, però, questi manufatti sono stati studiati in relazione alla scelta dei materiali e alle relative tecniche di realizzazione, né tantomeno ai conseguenti sviluppi della cultura artistica iberica d'età barocca, che in parte influenza ma viene soprattutto influenzata, dalle idee e dal gusto dei maestri italiani voluti da

Palacios. Lo studioso spagnolo ricorda, inoltre, che la passione per il collezionismo derivava da Filippo IV, e che Olivares fece costruire il Buen Retiro per ospitare le collezioni d'arte. Nelle stanze del Monterrey nel Palazzo Reale si trovavano originali di Tiziano e di Raffaello che egli stesso aveva acquistato a Roma e a Napoli, oltre al ricco vasellame d'argento, mentre la selezione per la sua biblioteca era stata affidata a Martín Miguel Navarro e Felice de Blanchis. (cfr. A. GONZÁlES-PALACIOS, "Un adornamento vicereale per Napoli", Civiltà del Seicento a Napoli, catalogo della mostra, Napoli, 1984, vol.II, pp. 241-302, pp. 241-252).

${ }^{6}$ D. DOMBROWSKI, cit., p.87.

${ }^{7}$ Cfr. : A. GonzÁles-Palacios, cit.; P. GIUsti, "Metalli", in Civiltà del Seicento.. cit, vol. II, pp., 326-335, p.328; P. D'AGOSTINO, cit. Nel suo recente contributo la D'Agostino attribuisce all'artista lombardo, attraverso un'attenta revisione bibliografica, un bronzetto barocco italiano del Metropolitan Museum di New York, un piccolo San Pietro che faceva parte in origine de1l'Altare maggiore della chiesa delle Agustinas di Salamanca. 
una committenza colta, cattolica e assolutamente devota. In questa direzione gli articoli citati del Prota-giurleo sono ancora un valido ed un utile punto di riferimento. La bibliografia spagnola esistente sull'argomento e che si è potuto consultare, inoltre, non nega che siano soprattutto i documenti degli archivi napoletani, redatti in lingua spagnola, a riferire informazioni significative sull'attivita' degli artisti italiani a Salamanca. Alcuni testi fondamentali sull'argomento aggiungono, però, alle conoscenze delle fonti napoletane notizie preziose dedotte dai documenti reperiti negli archivi spagnoli, in particolare nei fondi dell' Archivio Histórico de Protocolos di Madrid, della Parrocchia de San Sebastián di Madrid ed in particolare della Casa de $\mathrm{Alba}^{8}$. Queste fonti documentarie contribuiscono tutte a chiarire le esigenze dei mecenati ed i loro rapporti con gli artisti e con le comunita' religiose per cui questi edifici sacri vennero costruiti secondo le indicazioni progettuali e decorative italiane.

\section{UN COMMITTENTE D'ECCEZIONE: IL CONTE DI MONTERREY}

Nel testo che Angela Madruga Real dedica interamente alla chiesa e al convento de las Agustinas de Monterrey si ricostruisce attraverso un ampio e articolato percorso storico che va dal 1591, data dell'arrivo delle monache a Salamanca, al 1747, anno in cui si conclude il dilatato periodo della costruzione, la storia di una comunità religiosa, dei suoi problemi di assestamento, della sua relazione con la Cofradia di San Roque e dei suoi diversi domicili e ricoveri, che la accolsero fino al 1640, anno in cui le Agostiniane si trasferirono nel nuovo convento. A quest'ultimo il Monterrey destinò una posizione prestigiosa e strategica, di fronte al Palazzo di famiglia. Lo studio

\footnotetext{
${ }^{8}$ Cfr. A. MAdrUga Real, Arqitectura Barroca..., cit., p.14.
}

della Madruga Real evidenzia, inoltre, uno dei momenti più importanti del viceregno spagnolo per i rapporti culturali che il Monterrey intrattiene con gli artisti italiani, soprattutto napoletani. Il suo impatto con la città fu brusco e ricco di pathos se si tiene conto che fu proprio nella notte tra il 15 e il 16 dicembre del 1631 che Napoli visse la tragica vicenda dell'eruzione del Vesuvio e delle sue drammatiche conseguenze. $\mathrm{E}^{\prime}$ possibile che l'evento, raccontato con enfasi dal biografo Alonso de Contreras, capitano al suo servizio, turbasse non poco il viceré che ne fu cosi' spaventato e colpito che, per una subitanea maggiore consapevolezza della fragilità dell'esistenza umana, decise definitivamente di fondare una chiesa di famiglia; un luogo sacro che inteso ad ospitare una sorta di panteón per la sua sepoltura e per quella della moglie, donna Leonor, fosse concepito come un vano seminterrato che avrebbe dovuto sostenere a modi cripta il presbiterio?.

Fu sempre il ricordo ancora vivo della drammatica circoscostanza dell'eruzione a suggestionare e forse ad influenzare, pochi anni dopo, l'iconografia della tela commissionata al Ribera che nel 1635 rappresentò San Gennaro avvolto in una nube sullo sfondo del Vesuvio ${ }^{10}$ (Lám. 2). Il quadro che ancora oggi si trova sulla parete

\footnotetext{
${ }^{9}$ Si esclude che la decisione di fondare la nuova chiesa dipendesse dal fatto che Ines de Zúñiga (16401715), figlia naturale del Conte, avesse poi passato quasi tutta la sua vita nel convento salmantino delle Agostiniane. Le ragioni sembrano, invece, più verosimilmente legate alla volontà del committente di essere ricordato.

${ }^{10}$ IDEM, p.38 : "La primera obra directamente relacionada es probablemente el cuadro encargado a Ribera y que representa a San Genaro en una nube teniendo como fondo el Vesuvio, y la que culmina el proceso es desde luego la fundación de las Agustinas de Salamanca". In realtà come suggeriva Roberto Longhi non c'è alcuna relazione diretta tra quest'evento e la datazione del quadro del Ribera (cfr. nota 4). Tuttavia la lettura iconologica della Madruga Real è convincente, se non altro dal punto di vista dello stato d'animo del committente.
} 
sinistra della chiesa dentro una cornice di marmi policromi, fa parte, come già ricordava il Longhi, di una vera e propria pinacoteca strettamente connessa con il programma decorativo complessivo del presbiterio, affidato interamente al Fanzago che affianca, inoltre, in una stretta e duratura collaborazione Bartolomeo Picchiatti (Ferrara 1571 circa-Napoli 1643), ricordato come l'ingegnere o l'architetto napoletano, nella progettazione della chiesa. Se l'incarico ufficiale al Picchiatti è giustificato dal fatto che fosse stato nominato nel 1628 Ingegnere maggiore del regno, carica che mantenne fino al $1643^{11}$, occupando una posizione prestigiosa di comando a cui il committente non si poteva mostrare indifferente, fu Cosimo Fanzago il regista che realmente incise sul disegno della parte più importante della chiesa $^{12}$. Ma né il Fanzago né il Picchiatti, né tantomeno Giuliano Finelli (Carrara 1601 o 1602 - Roma 1653) che scolpisce i due splendidi ritratti funerari del viceré e di sua

11 "Si tratta, come noto di una delle cariche più autorevoli e meglio remunerate del tempo, in quanto al titolare veniva concesso "a vita" l'ufficio che gestiva gran parte delle opere di architettura e di ingegneria finanziate dalla Regia Corte. (...) dalla fine del Cinquecento e nel corso del Seicento la citata nomina era stata conferita a Domenico Fontana (1593-1607), al figlio Giulio Cesare (1607-1627), a Bartolomeo Picchiatti (1628-1643) e a Onofrio Antonio Gisolfo. Quest'ultimo nominato nel 1643, avrebbe ricoperto l'incarico fino al 1656, anno della sua morte e quindi gli sarebbe successo Francesco Antonio Picchiatti, figlio di Bartolomeo(1656-1694). (1) Ma fonti documentarie coeve rinvenute recentemente nell'archivio di Stato di Simancas, almeno per quanto concerne il Gisolfo, e di conseguenza il suo successore, contrastano con quanto sostenuto sinora dagli studiosi. Da esse, infatti, emerge che il Gisolfo, in seguito ad una complessa vicenda, fu sostituito nella citata carica da Cosimo Fanzago, probabilmente intorno al 1647."( Cfr. F. A. FIADINO, "Cosimo Fanzago Ingegnere maggiore del Regno di Napoli e la sua attività nel Palazzo Reale(1649-1653)", in Opus. Quaderno di Storia dell'Architettura e Restauro, 6, 1999, pp. 351-376, p.351).

${ }^{12}$ Cfr. A. MAdruga ReAl, Arquitectura Barroca..., cit., pp. 53-106. Cfr. pure P. D'AGOSTINO, cit., p.87, nota 10. $\operatorname{moglie}^{13}$, si recarono mai a Salamanca, e tutti i progetti e molte opere furono inviati in Spagna dalla Capitale del Regno. Fu, invece, all'architetto Curzio Zagarella, l'unico artista tra quelli assoldati dal Monterrey a Napoli a vivere nella città spagnola (dalla fine del 1635 al 1639 circa), che venne affidata la direzione dei lavori della chiesa, compito in cui fu seguito da artisti spagnoli del calibro di Francisco de la Hoya, García de Haro, Bartolomé Zumbigo ${ }^{14}$. E si deve proprio a questa serena collaborazione tra differenti personalità, il risultato originale e unico di quest'opera, che è stata giudicata una perfetta sintesi di elementi spagnoli e italiani, dove convivono, sotto lo sguardo vigile e sollecito del commitente, il dinamismo creativo di Fanzago che oscilla ancora tra tradizione tardo-manierista del portale maggiore e novità nell'arredo fantasioso polimaterico e policromo, il barocco desornamentado y austero di Gomez de Mora (Chiostro del Convento), le idee pratiche di Fray Lorenzo di San Nicolás che si occuperà della nuova cupola $(1675-1679)^{15}$.

Ma il rapporto del Vicerè con il Fanzago fu certamente privilegiato rispetto agli altri, se ormai si puo' affermare che sono le

${ }^{13}$ Sui ritratti funerari di Don Manuel e Donna Leonor scolpiti dal Finelli tra il 1636 e il 1637 cfr. pure : A. NAVA CELlinI, cit., p.40; G.G. BORRELLI, "Note per uno studio della scultura funeraria a Napoli nel Seicento", La scultura del Seicento.., cit., pp.145-167, p.154155. Ringrazio, inoltre, il mio amico e collega Dott. Roberto Alonso, per avermi segnalato una recentissima comunicazione di ALFONSO ROdRíGUEZ DE CEBALLOS Y RAQUEL NOVERO PLAZA intitolata "La rapresentación del poder en monumentos funerarios del barroco español: los sepulcros de los condes de Monterrey en las Augustinas Descalzas de Salamanca" del convegno "Arte, poder y sociedad en la España de los siglos XV al XX", tenutosi nel Istituto de Historia (CSIC) di Madrid dal 20 al 24 Novembre 2006 i cui atti sono in corso di pubblicazione.

${ }^{14}$ Cfr. A. Madruga ReAl, Arqitectura Barroca..., cit., pp.60-61.

${ }^{15}$ Cfr. IDEM, pp.165-166. Nel 1747 verrà posta sulla nuova cupola la veleta di metallo.(IVI, p.102). 
sue idee a prevalere anche sul progetto della chiesa. Di indubbia cultura architettonica italiana, ancora rispettosa dei dettami controriformistici, la chiesa del Monterrey non assomiglia per niente alle altre chiese castigliane seicentesche. Di nuova fondazione, e dunque libera da quelle stratificazioni che invece avevano caratterizzato molti degli edifici sacri medievali o rinascimentali nel XVII secolo, dato ancor più significativo in un luogo come Salamanca dove con straordinaria continuità nel segno architettonico e nell'uso dei materiali e nel gusto decorativo la Catedral Nueva si accostava a quella Vieja, la chiesa presentava la novità di una pianta unica piuttosto ampia con cappelle laterali, crociera e presbiterio, in cui si apre la Capilla Mayor dove emerge sulla parete di fondo il capolavoro della chiesa, quello che $\mathrm{e}^{\prime}$ stato definito il più importante retablo di Spagna.

\section{DA ROMA A NAPOLI A SALAMANCA}

Pur essendo ancora rigoroso nell'impostazione geometrica e nella composizione speculare delle parti, il retablo dell'Altare maggiore presenta una struttura unitaria ma variamente articolata, e nell'uso di linguaggi diversi e nella ricchezza policroma dei materiali lapidei e delle relative tecniche artistiche impiegate. Emblematica a riguardo è la lettura che ne fa Gómez Moreno nel Catalogo monumental de Salamanca : "El retablo principal, con sus columnas corintias y caprichoso remate de escultura, es excelente para Hacer resaltar los cuadros que atesora sin distraer con su masa; (...)"16.

Così lo aveva descritto, invece, Fray Alonso de Villerino, cronista dell'ordine delle Agostiniane, nel 1691 “ (...) y todo el Retablo tiene su asiento en quatro Escudos que comiençan desde el suelo, tan primorosos en piedras, que parecen espejos, la moldura de la primera grada del Altar Mayor, es de mármol

\footnotetext{
${ }^{16}$ M. GÓMEZ MORENO, cit., pp. 295.
}

blanco, y ella de jaspe verde, la segunda es de lo mismo, y en la delantera tiene a trechos unos lazos de bronce dorado, y sobre esta están siempre dos ángeles de bronce dorado, $y$ dos hechuras de San Pedro, y San Pablo que acompañan la Custodia, la qual tiene su asiento en el Altar Mayor, y a raiz del esta la puerta del primer Sagrario, que es de bronce dorado, y gravado el Dulce Nombre de Jesús: tiene mucho adorno de piedras, y toda es de jaspes de diverso colores, con guarniciones de bronce dorado debaxo del segundo Sagrario ay un corredorcito con valaustres de bronce dorado, muy pulidos, y la puerta dél es de una piedra que llaman lapislacio, en los lados ay unas como capillitas de una piedra negra, y están arrodillados dos ángeles, uno a cada lado, que los coronan dos lazos de bronce dorado; tiene ocho columnas de a tercia, de una piedra de gran precio, y no se sabe su nombre, mas no se ignora, que dos de ellas de lance costaron cinquenta mil reales; tiene ocho jarritas de Agata, con sus asas, y remates de bronce dorado, y ramilletes de plata; corona esta pieza un modo de cúpula de lapislacio, labrada primorosamente, a modo de pecho de azero, con sus encaxes de bronce dorado en cada concha con grande gracia, y sus divisiones de alto a baxo; remata esta media naranja la cruz con su asiento de bronce dorado, muy conforme en el primor a todo lo demás"17. Disegnato dal Fanzago che è anche l'autore delle quattro statue delle nicchie superiori della Maddalena, della Madonna, di San Giovanni Evangelista, di San Giacomo Maggiore, e del Crocifisso, è soprattutto un capolavoro di scultura, ma anche di sintesi linguistica; ne impreziosisce la mensa d'altare il decantato Tabernacolo o Sagrario, opera certa di Cosimo, documentata e realizzata a Napoli nel 1636 e poi messa in opera a Salamanca molti anni più tardi, nel 1686, dagli argentieri Juan de

${ }^{17}$ F. AlONSO De VILLERINO, Esclarecido solar de las religiosas recoletas de nuestro padre San Agustín y vidas de las Insignes hijas de sus conventos, Madrid, 1691, Tomo II, p.5. 
Figueroa y Pedro Benitez ${ }^{18}$ (Lám. 3). Il García Boiza che lo assegna, come opera che dice documentata senza però esplicitarne la fonte, al platero Béjar Juan Bautista Chapuz, e ne copia la minuziosa descrizione che già nel 1691 ne fa Padre Villerino ${ }^{19}$, prima riportata, riferisce che il tabernacolo era stato, inverosimilmente, assegnato a Jacometrezo, cioè all'italiano Jacopo da Trezzo (Milano 1519-1589), chiamato a lavorare in Spagna da Filippo II, artefice delle figure di quello della chiesa dell'Escorial, che contava ben tredici statuette di metallo, e della custodia più piccola che si trova all'interno, in oro, argento e altri metalli, e pietre preziose, considerato in Spagna e non solo, un capolavoro nel genere ${ }^{20}$.

Realizzato, come anche altre fonti storiche documentano, con una varietà di materiali preziosi che Alonso de Villerino ebbe cura di elencare, bronzo dorato, lapislazzuli, diaspri di diverso colore, agata, argento, una pietra nera e una di gran pregio di cui non si conosce il nome, era in origine completato da due angeli e dalle due statuine di bronzo dorato di San Pietro e di San Paolo, poi scomparse. Il San Pietro, fra l'altro, è stato recentemente ritrovato da Paola D'Agostino e ricondotto all'opera di Fanzago per l'altare maggiore salmantino ${ }^{21}$.

\footnotetext{
${ }^{18}$ Sul retablo e sul tabernacolo cfr. A. MADRUGA REAL, Arqitectura Barroca..., cit., pp.126-129. Qui l'autrice attribuisce il disegno del retablo e delle sue parti interamente al Fanzago basandosi principalmente sui documenti pubblicati dal Protagiurleo nel 1957 e nel 1986 più volte citati.

${ }^{19}$ Cfr. : A. GARCÍA BOIZA, cit., pp. 27-28.

${ }^{20}$ Sul tabernacolo dell'Escorial si veda : R. COPPEL AREIZAGA, “Obras Realizadas en Bronce. Siglos XVXIX", Summa Artis. Historia General del Arte, Madrid,1999, Vol. XLV, pp.139-183, p.158.

${ }^{21}$ P. D'AGOSTINO, cit.. Qui la D'Agostino tra le fonti citate riporta anche le decrizioni del tabernacolo del Ponz (1783) dove le statuine sono ancora due e del Falcon(1867) dove sono già diventate quattro per l'aggiunta di due copie, come viene riportato anche da Gómez Moreno nel Catalogo monumental de Salamanca (1901). Se ne deduce che alla fine del '700
}

In relazione ai materiali e alle tecniche utilizzate il Gómez Moreno parla di un basamento todo de incrustaciones e definisce il sagrario un gioiello di valore impreziosito da lapislazzuli, malachite, diaspri, e modanature e decorazioni di bronzo dorato ${ }^{22}$. La Madruga Real, infine, nella sua descrizione della custodia allo stato attuale, scrive che oltre al bronzo dorato (angioletti, balaustra, porta del primo sagrario, e varie decorazioni) i materiali maggiormente impiegati sono il diaspro (primo basamento adornato dagli scudi del Monterrey in bronzo), il marmo rosso (doppio basamento su cui si elevano le colonne salomoniche), il lapislazzuli abbinato all'opale (parte centrale del primo registro poligonale, dove l'opale è incrostato col bronzo, e parte superiore del tamburo e della cupola), il marmo nero (loggette laterali) ${ }^{23}$. Emerge con evidenza da quanto detto finora, la sfortuna conservativa, soprattutto ottocentesca, di questo tabernacolo, come pure di altri manufatti barocchi dello stesso genere dell'Italia meridionale, a cui vanno imputate le sparizioni, le aggiunte, i cambiamenti, e una frequente disattenzione nel curare e preservare i materiali e le relative tecniche artistiche originali.

Dalla fine del ‘ 500 fino al ' 700 si assiste, infatti, in alcune aree del Mediterraneo occidentale come la Spagna ed in particolare in alcune regioni del Sud d'Italia come, ad esempio, la Campania, la Calabria e anche la Puglia, ad un'ampia diffusione dei tabernacoli, tipologia di arredo presbiteriale

l'Altar Mayor, le sue parti originarie e probabilmente i suoi materiali fossero ancora correttamente conservati. Più difficile è stabilire se gli angeli di bronzo dorato citati dal Villerino siano gli stessi ricordati dopo le cuatro estatuitas dal Gómez Moreno : "Dos ángeles arrodillados, de mayor tamaño, pero de la misma materia y estilo, estan puestos a los lados del tabernáculo".(cfr. M. GÓMEZ MORENO, cit., p.296).

${ }^{22}$ Cfr. M. GÓMEZ MORENO, cit., p.295.

${ }^{23}$ Cfr. A. MAdruga Real, Arqitectura Barroca..., cit., pp.128-129. 
destinato alla custodia delle specie eucaristiche e all'esposizione perpetua del Ss. Sacramento, decretato nella Chiesa Cattolica dal Concilio di Trento. Questo avviene anche grazie alla conoscenza e alla circolazione di alcuni modelli eccellenti disegnati e/o realizzati da artisti di provenienza e formazione tosco-romana, lombarda e padana, alcuni attivi nei cantieri architettonici più importanti romani e spagnoli nell'età della Controriforma ${ }^{24}$. Questi modelli circolano non solo per il tramite degli artisti in continua mobilità nei cantieri che vantano prestigiose committenze, ma anche attraverso $i$ disegni e le incisioni della stampa di settore, i trattati, le guide per gli stranieri, alcune già in formato tascabile.

Più che all'esemplare dell'Escorial, di cui forse emula la ricchezza delle pietre utilizzate e principalmente la profusione dei metalli, che richiedono una notevole sapienza esecutiva ${ }^{25}$, questa custodia va

\section{-}

${ }^{24}$ Sull'argomento, che fa parte integrante della ricerca che sto conducendo sulle arti decorative in metallo nel XVII e XVIII secolo nell'Italia meridionale nei suoi rapporti con il Mediterraneo occidentale, ed in particolare sui cibori campani ed i loro modelli, segnalo solo le fonti storiche e i contributi utilizzati per la stesura del presente articolo : C. DE LELLIS, Parte seconda o vero supplimento alla Napoli sacra di Cesare D'Engenio Caracciolo, Napoli, 1654; P. SARNELLI, Guida de Forestieri, Napoli, 1697; D. A. PARRINO, Napoli città nobilissima, antica e fedelissima, Napoli, 1712; G. SIGISMONDO, Descrizione della città di Napoli e i suoi borghi, Napoli, 1788, voll. I e II; L. GUARINI, "Descrizione della chiesa de' SS.Apostoli", Archivio Storico Italiano, IX, 1846; C. CELANO, Notizie del bello, dell'antico e del curioso della città di Napoli, ediz. a cura di G.B.CHIARINI, Napoli, 1856, vol.II e III; G. A. GALANTE, Guida sacra della città di Napoli, Napoli, 1872, ed. a cura di N.Spinosa, Napoli,1985; F. STRAZZULlO, La chiesa dei SS. Apostoli, Napoli 1959; A. LIPINSKY, "La chiesa metropolitana di Capua ed il suo tesoro", Archivio Storico di Terra di Lavoro, III, 1960-64, pp.348-355, pp.349-350; P. GIUSTI, cit.; A. GONZÁLES PALACIOS, cit.; S. F. OsTROW, L'arte dei papi. La politica delle immagini nella Roma della Controriforma, Roma 2002.

${ }^{25}$ Un altro tabernacolo spagnolo di uguale fama ed importanza realizzato in argento alla fine del '500 è fatta discendere, invece, secondo me, principalmente per la sua forma e per la sua struttura a tempietto, da un modello di custodia italiana, di ascendenza romana, di cui il prototipo potrebbe essere quella di San Giovanni in Laterano. Ma un modello eccellente nello sviluppo tipologico e funzionale per i cibori seicenteschi dell'Italia centro-meridionale fu senz'altro il monumentale Tabernacolo del Sacramento progettato da Domenico Fontana per la Cappella Sistina (1585-1590) in Santa Maria Maggiore, concepito come un modello di tempio ottagonale a due piani (quasi due metri di altezza) coronato da una cupola, tutto di bronzo dorato con intarsi di marmo colorato, e sostenuto al disopra dell'altare, a circa m 1,75 di altezza, da quattro angeli classicheggianti anch'essi in bronzo dorato, di due metri di altezza circa ciascuno ${ }^{26}$. In effetti la pianta centrale di matrice classicista e la struttura a tempietto furono molto in auge fin dal primo Seicento a Napoli. Mi riferisco in particolare ad alcuni cibori napoletani come, per esempio, quello della Chiesa della Ss.Trinità delle Monache, poi andato perduto insieme ad altri oggetti dopo la soppressione del 1806, commissionato al fiorentino Giacomo Lazzari nel 1621, preferito al senese Domenico Montini di cui furono, invece, rifiutati i bozzetti ${ }^{27}$. Questo ciborio assomigliava nella struttura più compatta e più sviluppata in larghezza, e per le dimensioni più contenute, a quello commissionato allo stesso Montini nel 1618 per l'antico altare dell'Annunziata rifatto su disegni del Fanzago, che il De Lellis stima "di bellissima architettura, con un ricchissimo baldacchino di rame indorato, con dei

senz'altro quello della Cattedrale di Siviglia firmato dal grande maestro Juan De Arfe.

${ }^{26}$ Cfr. S.F. Ostrow, cit., p.51.

${ }^{27}$ Sul tabernacolo della chiesa della SS.Trinità delle Monache cfr. : F. STRAZZULLO, "Schede per Giuliano Finelli, G. Mencaglia, G. e D. Lazzari”, Il Fuidoro, 4, 1957, pp.142-145, p. 144; F. StrazzUllo, La Chiesa dei SS.Apostoli, cit., p.64. 
Angiolini che dimostrano di sostenerlo", e che è l'unico oggetto parzialmente superstite dell'incendio del 1757, ma anche a quello, disegnato dallo stesso bergamasco, per la chiesa di Santa Patrizia (1619 ca.-1624), oggi a Capodimonte ${ }^{28}$ (Lám. 4). Sintesi esecutiva tra virtuosismi tecnici e linguaggi ornamentali di matrice tardo-manierista principalmente toscana, e prime invenzioni di una decorazione plastica naturalistica, quest'ultimo è a sua volta vicino, anche per alcuni motivi decorativi quasi identici come quello del pannello centrale con il vaso da fiori, al ciborio del 1619 di Domenico Montini, notevole per la ricchezza dei materiali utilizzati e anche per le sue dimensioni ( $\mathrm{cm} 189 \mathrm{x}$ $83 \times 60$ ), e conservato nel Palacio Real di Madrid. Costruito con pannelli e colonne in pietre dure, é decorato, similmente alla custodia de las Agustinas, con statuette a tutto tondo in bronzo dorato inserite nelle nicchie del corpo centrale e poste sulle cornici mistilinee ed aggettanti del tamburo e della cupola, e sul lanternino ${ }^{29}$ (Lám. 4). Se esiti monumentali di questa tipologia fanzaghiana si avranno in quello commissionato all'artista nel 1631 per la Certosa di Santo

${ }^{28}$ Sul ciborio dell'Annunziata cfr.: C. DE LELLIS, cit., p. 194; G. SIGISMONDO, cit., tomo 2, p. 137; C. CELANO, cit.,vol.3, p.830; F. STRAZZULLO, Schede per ..., cit., p.144; A. GONZÁlES PALACIOS, cit., p.261; U. PROTA GIURleO, "Cosimo Fanzago", cit., pp.22-23. Qui si apprende che i lavori per il baldacchino dorato da sovrapporre all'altare maggiore della chiesa, disegnato dal Fanzago, furono commissionati ad artefici diversi, tra cui Raffaele Maitener. Cfr.pure U. PROTA GIURLEO, "Fanzago ignorato" , cit., p.150, nota1, dove si rende noto il documento datato 18 settembre 1640 che attesta a Raffaele Maitener, tedesco della città d'Hispurgh, statuario di metallo, la commisione per l'opera in rame in getto ed in piastre per l'Altare maggiore della chiesa della SS.Annunziata, su disegno di Cosimo Fanzago.

Sul ciborio di Santa Patrizia, invece, cfr. : A. SPINOSA, I Cibori fanzaghiani di Santa Patrizia e dell'Addolorata di Serra San Bruno, Napoli, 1974; C. FIORILlo, “Omaggio a Cosimo Fanzago : il ciborio per Santa Patrizia", Napoli Nobilissima, 4 ser., 31, 1992, pp.87-96.

${ }^{29} \mathrm{Su}$ questo tabernacolo del Montini confronta la descrizione accurata che ne fa A. GONZÁles PALACIOS, cit., p.245 e pp.261-264.
Stefano del Bosco in Calabria, ora nell'Addolorata di Serra San Bruno, alcuni elementi naturalistici di precoce invenzione torneranno in miniatura nella custodia salmantina; e ne sono un esempio evidente $\mathrm{i}$ sei angioletti in bronzo dorato che decorano il tamburo e la cupola e i due inginocchiati che si affacciano dalle loggette laterali del registro centrale ${ }^{30}$. Per realizzare le parti in metallo il Fanzago si avvalse, così come fece per altre opere importanti tra cui il ciborio di Serra San Bruno, della collaborazione di Raffaele Maitener detto il Fiammingo o il Tedesco, allora attivo a Napoli in numerosi cantieri (San Paolo Maggiore, Ss. Apostoli, S. Trinità delle Monache, Santa Patrizia), "abilissimo gettator di metalli e specialista nel costruire custodie", come il maestro da cui andò a bottega, il milanese Bartolomeo Bertaglia $^{31}$.

Così, anche nel disegno del presbiterio della chiesa salmantina ritorna quella cultura artistica e decorativa unitaria che non separa il progetto architettonico dal suo arredo, e fa della scelta policroma dei materiali e dell'uso dei differenti linguaggi

${ }^{30}$ Sul ciborio di Serra San Bruno cfr. : A. SPINOSA, I cibori fanzaghiani.., cit..; M. PANARIELLO, "Il tabernacolo dell'antico altare della certosa", Daidalos, 2001, n.1, pp.60-65. Un ulteriore sviluppo monumentale si ebbe nel ciborio del Fanzago per la Cattedrale di Palermo (1653-58) su cui il Garstang scrive : "Non ignoto a Napoli, questo tipo di custodia di derivazione romana gode di particolare favore a Palermo dal tardo Cinquecento fino all'inizio del Settecento" (D. GARSTANG, "Cosimo Fanzago e le maestranze palermitane", Civiltà del Seicento..., cit., pp.387-401, p.390).

${ }^{31}$ Cfr. U. ProtA-GIURLeo, "Fanzago ignorato", cit., p. 150 e IDEM, "Cosimo Fanzago", cit., pp.21-23. Bartolomeo Bertaglia noto con il nome di Bartolomeo Viscontini, fu attivo a Napoli dal 1612 circa. Egli stesso in un documento del 1632, in cui si definisce scultore e fonditore specializzato in custodie, racconta che lasciata milano all'età di 21 anni, si trasferisce definitivamente a Napoli, dopo un soggiorno romano di circa un anno presso la corte papale di Paolo V, dove lavora ai tabernacoli di S.to Liguoro, dei Ss.Apostoli, della Ss.Annunziata. (Cfr. F. StrazzUllo, La Chiesa dei SS.Apostoli, cit., p.76). 
un elemento di spinta innovativa, inaugurata dal Bernini e mai abbandonata dall'artista lombardo fin dai tempi della Certosa di San Martino.

\section{LA PERDUTA REJA DEL FANZAGO}

Delle opere realizzate in metallo che completavano l'arredo presbiteriale de Las Agustinas le fonti storiche spagnole sono povere, se non del tutto prive, di riferimenti puntuali. Tranne che per il Tabernacolo che, come abbiamo visto, viene descritto con dovizia di particolari, non si sono rintracciati accenni alla cancellata monumentale che chiudeva il presbiterio.

Eppure, in uno dei documenti del fondo napoletano del notaio Andrea Fasano scritti in lingua spagnola si legge che il Cavallero Cosme Fanzago escultor si impegna a realizzare oltre al retablo in marmo per l'altare maggiore della cappella delle Monache di Sant'Orsola in Salamanca (primo luogo di destinazione dell'opera) con le cinque figure del Crocifisso, della Madonna, di San Giovanni Evangelista, di Santiago Maggiore e della Maddalena, anche "dos apoyadores para Rejas" ${ }^{32}$. Queste cancellate poi scomparse avrebbero dovuto appoggiarsi evidentemente, come descritto dal documento citato, che reca la data 28 novembre 1633 ed è dunque precedente all'inizio della costruzione della nuova chiesa de Las

\footnotetext{
32 Il documento è riportato in U. PROTA-GIURLEO, "Fanzago ignorato", cit., p.147 e in IDEM, "Cosimo Fanzago", cit., p. 19. In realtà in esso si fa riferimento all'idea primitiva del Monterrey di ubicare il Retablo in una cappella nella Chiesa di Sant'Orsola (giudicata poi forse troppo piccola per accogliere un'opera così concepita che richiedeva uno spazio adeguato dove essere collocata) prima che il viceré decidesse di edificare la nuova chiesa nel 1635, dove desiderava essere sepolto insieme alla moglie. Cosa che in realtà non avvenne poiché quando nel 1653 il Conde mori' (e dopo un anno sua moglie), la realizzazione del panteón non era stata portata a termine per i numerosi e continui problemi legati alla profonda umidità del terreno.
}

Agustinas (1635), sulle due transenne o balaustre che oggi occupano un posto diverso da quello originario e sono poste ai lati della cappella dell'altare maggiore (Lám. 5). Esse sono identiche ed esibiscono una decorazione barocca di veri e propri ricami marmorei, secondo la Madruga Real "un perfecto trabajo de calado semejante al del apoyador que hay en la iglesia de la Cartuja de San Martin" ${ }^{33}$, e si differenziano profondamente nel gusto dalle due grate di ferro battuto, essenziali e severe, sotto le quali attualmente sono poste. Delle due grate il Padre Villerino cita quella del Coro nella conclusione della sua descrizione dell'Altare Maggiore e a proposito delle due statue dei Fondadori del Finelli : "Sobre la rexa del Coro, y la que le corresponde están en sus nichos los retratos, y vultos de los señores Fundadores arrodillados, de estatura natural de mármol blanco, y delante ay unos bancos con sus respaldo en el suelo de diversos colores de jaspes muy labrados, y unos embutidos que causan grande admiración: las gradas por donde se sube al Altar mayor son seis, todas de mármol pardo $^{\prime 34}$. Le gelosie che sono simili nel disegno e nella tecnica esecutiva a quelle delle finestre del convento della chiesa, lasciano intravedere i due ambienti a destra del locutorio e a sinistra del coro che, come si legge in un altro documento spagnolo, vengono costruite nel rispetto della legge, secondo l'obbligo già controriformista di chiudere le cappelle e i luoghi di clausura con cancelli e grate ${ }^{35}$. Ma la ricostruzione della cappella dell'altare maggiore va completata seguendo le indicazioni del documento che descrive la cancellata che la chiudeva, di ferro battuto con ornamenti di

\footnotetext{
-

33 Cfr. A. MAdruga ReAl, Arquitectura barroca.., p.130.

${ }^{34}$ F. AlONSO DE VILLERINO, cit.

${ }^{35}$ Cfr. A. MAdRUGA ReAL, Arquitectura barroca.., cit., p.180. Per l'accostamento tra le due grate del presbiterio e quella di una finestra del convento annesso alla chiesa cfr. A. GAllego DE Miguel, Rejeria castellana. Salamanca, Salamanca, 1977, p.122.
} 
ottone. Il documento redatto dinanzi al notaio Andrea Fasano di Napoli il 4 gennaio 1635 e al mastro ferraro Giulio d'Ambrosio e al Cavalier Cosme Fanzago riferisce su l'impegno del D'ambrosio, herrero, a procurare il "ferro labrado, seys quintales en circa por tiempo de quatro meses, conforme el mudelo que se dara .....", materiale necessario per la realizzazione della reja richiesta dal Monterrey per la Capilla de la Concepción de Salamanca, e descrive più avanti il disegno delle due porte di metallo che nel progetto del Fanzago costituivano la cancellata, "de peso de libras dos mill ciento $y$ veynte $y$ dos", che si appoggiava sulle transenne marmoree, con le cornici e i balaustrini in ottone, le decorazioni floreali tra un balaustrino e l'altro e quelle poste sopra la cornice superiore in metallo, cioè in ferro battuto $^{36}$. La reja fu completata in un anno, se in un documento rogato dallo stesso notaio il 28 febbraio 1636, tra i lavori già eseguiti dal Fanzago, vengono elencati " $\mathrm{La}$ custodia de Metalo de lapis lazaro, Retablo de pasitos, losa de tierra, Reja, Apoyador, Estatua de marmol, y otras obras contenidas ..." ${ }^{\prime 37}$. Il cancello monumentale, poi forse distrutto e comunque scomparso, viene ricordato anche da Gonzáles-Palacios che lo cita in relazione al disegno (1625-1650 ca.) conservato nel lascito Blunt del Courtauld Institute di Londra; disegno che, secondo lo studioso, potrebbe essere una delle idee progettuali del Fanzago per la costruzione di cancelli monumentali, come quello di Salamanca, e non necessariamente un primo disegno preparatorio per la Porta del Tesoro di San Gennaro del Duomo di Napoli ${ }^{38}$ (Lám. 6 ).

\footnotetext{
${ }^{36}$ Cfr. : U. PROTA-GIURLeO, "Fanzago ignorato", cit., p.148 e in IDEM, "Cosimo Fanzago", cit., p. 19.

${ }^{37}$ Cfr. : IDEM, "Fanzago ignorato", cit., p.148. Nello stesso periodo furono commissionati al Fanzago altri lavori di marmo, tra cui l'ornamento d'altare per la tela del San Gennaro del Ribera e il pulpito.

${ }^{38}$ Cfr. P. GIUSTI, cit., p.328. Sui cancelli del Fanzago a Napoli e sulla diffusione dei suoi modelli nell'Italia meridionale si veda il mio recente articolo :
}

Si ricordi, inoltre, che a Salamanca dove nella prima metà del secolo XVII, si stava sperimentando un nuovo linguaggio barocco in opere come la Clerecia de los Jesuitas, la sagrestia di San Esteban, la Cattedral Nueva, in quello stile semplice e austero, che derivava in ultima istanza dalle forme escurialensi, la chiesa del Monterrey diventa il manifesto del primo barocco napoletano. E uno degli elementi importanti che gli conferiscono il carattere italiano è l'impiego dei materiali. In effetti tutti i marmi utilizzati nell'esecuzione furono inviati da Napoli nel 1638; da Cartagine, dove arrivavano con le navi, venivano trasportati prima a Madrid e da lì con i carri a Salamanca. Oltre ai marmi, come recitano i documenti, furono trasportate altre casse con varie cose e una reja de bronce, forse la stessa di cui sopra abbiamo riportato la descrizione ${ }^{39}$. Spesso, infatti, le carte d'archivio commettono errori grossolani nell'indicare i materiali utilizzati nei manufatti in metallo, confusioni dovute alla scarsa conoscenza delle leghe ma anche di tecniche artistiche molto complesse, dalle fusioni a cera persa e a staffa del bronzo e dell'ottone, all'imbutitura del rame, allo sbalzo dell'argento. Fin dal Medioevo, infatti, nella rejeria spagnola, era prevalso l'uso del ferro (battuto, forgiato, fuso, sbalzato, cesellato, dipinto e dorato), a cui fin dalla seconda metà del '500 era stato in alcuni casi accostato o sostituito del tutto il legno, spesso di qualità pregiata, per ragioni economiche legate alla committen$\mathrm{za}$, ma anche per assonanza con l'ampia diffusione dei retablos ${ }^{40}$. La fortuna delle arti

M. BOzZI CORSO, "Sulle arti decorative in metallo tra Napoli e la Terra d'Otranto in età barocca. Segnalazioni e aggiunte", Ottant'anni di un maestro. Omaggio a Ferdinando Bologna, a cura di F. ABBATE, Napoli, 2006, tomo II, pp. 477-487.

${ }^{39}$ Cfr. A. MADRUGA REAL, Arquitectura barroca, cit., p. 61.

${ }^{40}$ Della vasta bibliografia spagnola consultata sugli artisti e le arti del metallo in genere e soprattutto sulla rejeria dal Medioevo all'età contemporanea, si 
decorative e della scultura in metallo italiana in Spagna in età barocca, inoltre, è più che nota. E non solo quella dei manufatti in argento, in oro e pietre preziose. Già dal '500 la statuaria in bronzo italiana si diffondeva in Castiglia, dall'arredo scultoreo e dalle raccolte di Palazzo Reale a Madrid alle statue monumentali di Pompeo Leoni, Los Entierros per la Basilica dell'Escorial e Los duques de Lerma. Queste ultime due, in bronzo dorato, straordinarie per la loro imponenza e per le raffinate e complesse tecniche d'esecuzione delle superfici, furono modellate in gesso dall'artista italiano e fuse da Juan De Arfe nel 1602 per la chiesa di San Pablo di Valladolid, ora esposte nella attigua cappella gotica del Colegio de San

segnalano solo i seguenti studi ritenuti indispensabili e strettamente pertinenti alla stesura di questo articolo : J.J. MARTin GONZALES, "La vida de los artistas en Castilla la vieja y Leon durante el siglo de oro", Revista de Archivos, Bibliotecas y Museos, LXVIII, 1, 1959, pp. 391-489; S. AlCOLEA, "Artes decorativas en la España Cristiana (siglos XI-XIX)", Ars Hispaniae. Historia Universal del Arte Hispanico, Madrid, 1975, vol. 20 ; A. Gallego De Miguel, cit.; F. De Olaguer-Feliu Y AlONSO, Hierro, Rejeria, in Historia de las artes aplicadas e industriales en España, Madrid, 1982, pp.17-64; IDEM, "Notas para una historia de la rejeria architectonica madrilena", Anales del Instituto de Estudios Madrilenos, XIX, 1982; A. GAllego De Miguel, "El arte del hierro en la Catedral de Burgos", Academia, 57, 1983, pp.213240; A. BARbero GarcíA, T. De Miguel Diego, Documentos para la historia del arte en la provincia de Salamanca, siglo XVI, Salamanca, 1987; A. BARBERO GARCíA, T. DE Miguel DIEGO, Documentos para la historia del arte en la provincia de Salamanca, siglo XVII, Salamanca, 1988; M. LUISA HERRERO GARCíA, “Los Elorzas. Una familia de rejeros vascos", Archivo Español de Arte (AEA), 248, 1989, pp. 459-465; M. L. HERRERO GARCÍA, Rejeria en Segovia, Segovia 1993; A. GAllego DE MiguEL, "Rejeria", Historia del Arte de Castilla y Leon, Valladolid 1997, VI, pp.437-454; IDEM, Rejeria castellana. Zamora, Valladolid, 1998; F. DE OLAGUER-FELIU Y ALONSO, "El arte del hierro en España. La forja monumental", Summa Artis, Madrid,1999, vol.XLV, pp.37-101; "Las artes decorativas en Europa", Summa Artis. Historia General del Arte, Madrid, 2000, vol.XLVII-I; J. MATESANZ, Actividad Artistica en La Catedral de Burgos. De 1600 a 1765, Burgos, 2001.
Gregorio che ospita il Museo Nacional de Escultura $^{41}$.

Di bronzo patinato sono, invece, i due angeli reggifiaccola o reggitorciera che completano l'arredo scultoreo del presbiterio, e che ancora oggi si trovano sui due pilastri laterali all'ingresso della Capilla Mayor, sulle due grandi volute turgide e scavate nel bronzo che fungono da mensola, gia' segnalati e messi in relazione con quelli quasi identici di Santa Maria la Nova, di bronzo dorato che, come dice il Celano, "col modello del Cavaliero furono tragittati con grandissima diligenza, da Raffaele il Fiammingo" e "situati sopra certi cartocci, che tengono le lampane pendenti dalle mani." ${ }^{42}$ (Lám. 7 e 8). Realizzate forse anch'esse in collaborazione con il Fiammingo, le sculture salmantine, per l'evidente naturalismo e per l'acceso plasticismo delle figure, sembrano avvicinarsi anche a quelle che lo stesso Fanzago disegna negli stessi anni, per esempio, per il ciborio calabrese di Serra San Bruno (Lám. 9).

Ma nonostante sia stato sottolineato quanto la congiuntura dei maestri italiani nella realizzazione de Las Agustinas sia stata importante per Salamanca, non si è finora tentato di ricostruire il ruolo che tale opera esercitò sullo sviluppo della cultura artistica spagnola del XVII e XVIII secolo. Eppure sopravvivono opere che possono essere lette come riflessi della presenza di un autentico capolavoro del Fanzago in città, oltretutto il primo esempio in assoluto di un conjunto pienamente barocco in Spagna.

L'imponente reja napoletana, unico esemplare italiano nella prima metà del secolo, ha dovuto di certo rappresentare un prototipo, un modello nuovo ed originale,

\footnotetext{
${ }^{41}$ Cfr. R. Coppel AREIZAGA, cit., p.156-157.

${ }^{42}$ Cfr. U. Prota-GiURleo, "Cosimo Fanzago", cit., 1986, p.23.
} 
se in alcuni cancelli barocchi della Cattedral Nueva di Salamanca, ma anche in quelli settecenteschi del coro della Cattedral di Ciudad Rodrigo, ritornano fra l'altro, anche se rielaborati e reinterpretati in contesti decorativi diversi, alcuni motivi geometrici che si avvicinano ai primi repertori figurativi fanzaghiani e alle loro evoluzioni formali di alcuni esempi napoletani. A Salamanca questa circolazione dei modelli è dovuta anche alla presenza nel cantiere de Las Agustinas di Gómez de Mora, artista sensibile e aperto agli stimoli del nuovo stile post-herreriano, che incide fortemente nella formazione del primer barroco dell'architettura spagnola, facendo senza dubbio da filtro ma anche da trade-union tra la cultura artistica italiana della chiesa del Monterrey e gli altri cantieri urbani. Se il secolo d'oro per la rejeria spagnola, ed in particolare per quella di Castiglia y Leon, resta senza alcun dubbio il XVI, quando si formano gli artisti e le scuole locali di maggior pregio e prestigio, i segni del cambiamento in età barocca vanno letti nell'importanza che nelle rejas monumentali assumono i balaustrini lisci e torniti, non più cesellati e decorati a sbalzo come quelli di alcune splendide cancellate rinascimentali, e i coronamientos, dove già dalla fine degli anni Venti riappaiono i motivi geometrici, le piramidi, le volute e le linee di matrice herreriana dell'opera di Gómez de Mora nella Clerecia de Los Jesuitas. Ne sono esempi evidenti nella Cattedral Nueva di Salamanca le rejas della Capilla de Nuestra Señora de la Verdad, e di quella di San Lorenzo, di cui ne troviamo quasi una copia nella Capilla del Licenciado Gonzales nella Chiesa di Fuentalpeña nella vicina Zamo$\mathrm{ra}^{43}$.

\footnotetext{
${ }^{43}$ Cfr. : A. Gallego De Miguel, Rejeria Castellana.Salamanca cit., p.120, 124, 126; IDEM, Rejeria castellana. Zamora cit., p.125.
}

Andrebbero invece indagati gli echi di alcune opere dell'artista italiano in altre inferriate barocche, già settecentesche, come, per esempio, alcune di quelle laterali del secondo corpo della capilla Mayor nella cattedrale di Segovia, realizzate da rejeros delle famose forjas basche tra il 1694 e il 1736. In esse compaiono su entrambi i lati dei coronamenti dai raffinati motivi floreali in ferro forgiato e dipinto, lavorate a tutto tondo, sculture lignee di figure di santi, sporgenti e rappresentati quasi per intero, en medio cuerpo, realizzate da Mateo Negrete e che ricordano, se non altro nella struttura compositiva, il prototipo del busto bifronte del San Gennaro in ottone del cancello del Tesoro del Duomo di Napoli ${ }^{44}$. (Lám. 10 e 11) Ancora legate ad un linguaggio solo decorativo, soprattutto per le loro ridotte dimensioni nella relazione proporzionale che assumono all'interno delle strutture metalliche, queste "piccole" sculture non hanno certo la forza espressiva ed il languore patetico del San Gennaro di Fanzago, ma anzi, forse sviluppano, in un linguaggio senza dubbio diverso e originale alcuni elementi figurativi di cancellate spagnole già rinascimentali.

\footnotetext{
${ }^{44}$ Cfr. M. L. HeRrero García, Rejeria en Segovia cit., pp.276-279.
} 


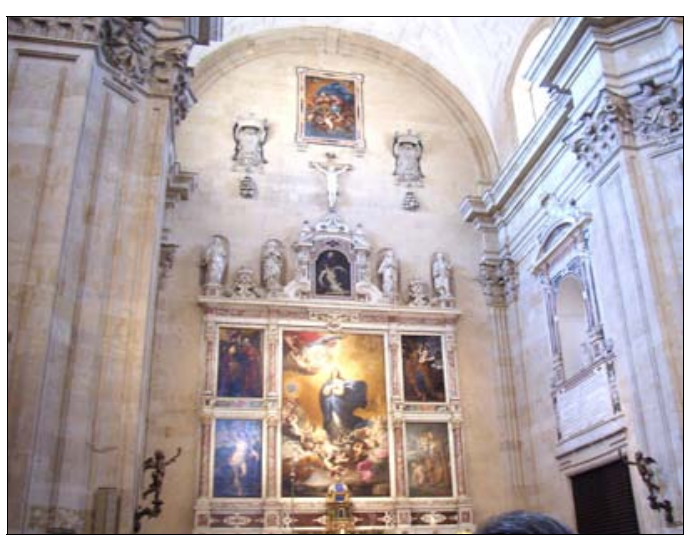

- Lám. 1. Altare maggiore con il retablo. Salamanca. Chiesa de Las Agustinas Recoletas. (Fotografia dell'autrice).

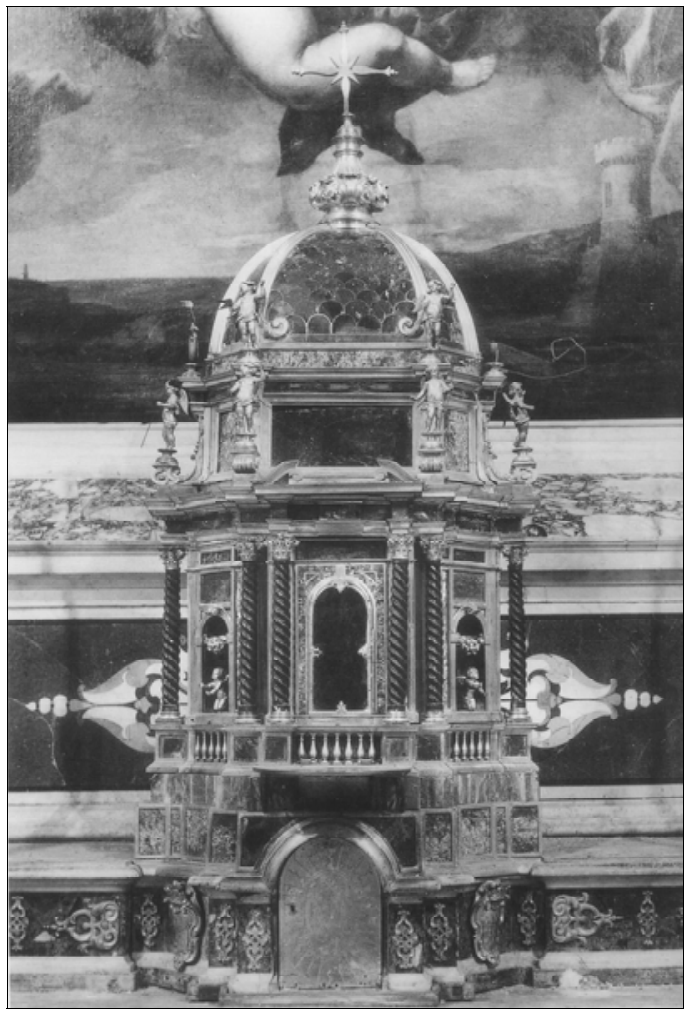

- Lám. 3. Tabernacolo dell'Altare maggiore. Salamanca. Chiesa de Las Agustinas Recoletas (da Prospettiva, 109, 2003 (2004)).

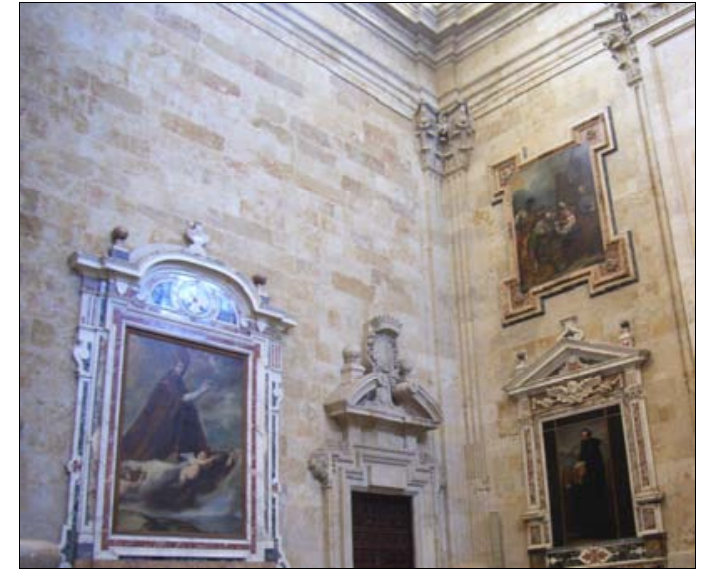

- Lám. 2. Ribera, San Gennaro. Salamanca. Chiesa de Las Agustinas Recoletas. (Fotografia dell'autrice).

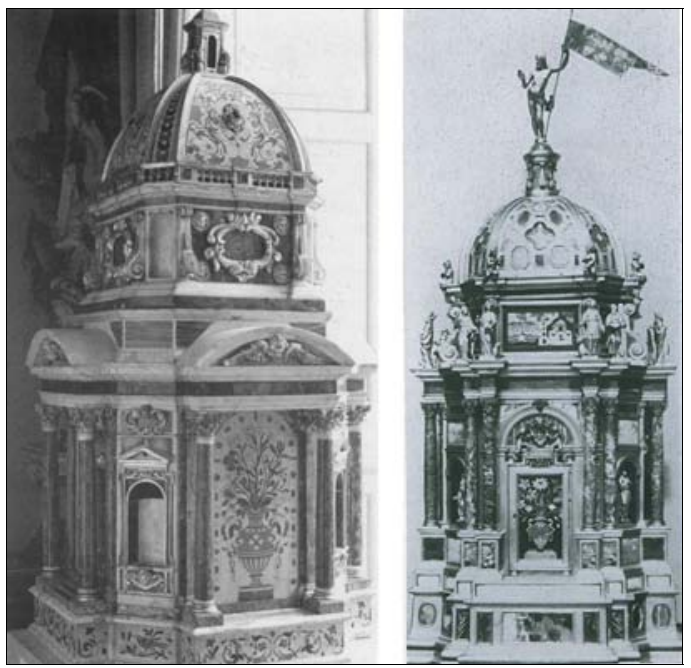

- Lám. 4. Ciborio della Chiesa di Santa Patrizia. Napoli. Museo di Capodimonte. (da $\mathrm{Na}$ poli Nobilissima, 4 ser., 31, 1992); Custodia. Madrid. Palacio Real. (da Civiltà del Seicento a Napoli, cat. della mostra, Napoli, 1984, vol. II). 


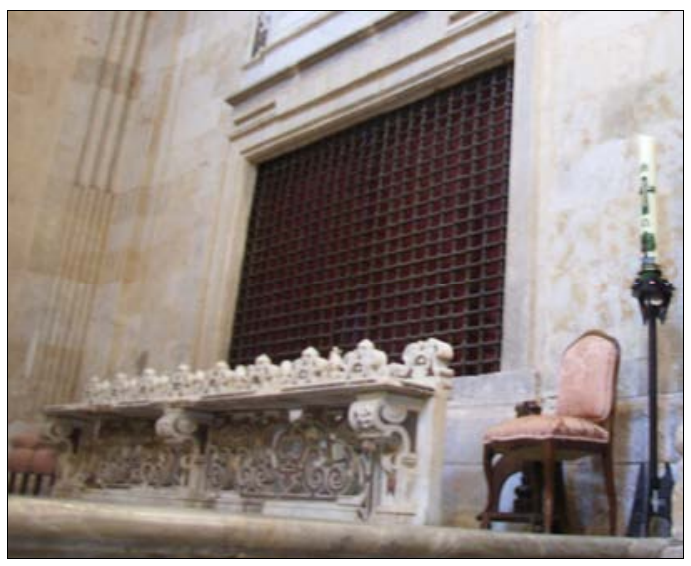

- Lám. 5. Grata del coro con apoyador. Salamanca. Chiesa de Las Agustinas Recoletas. (Fotografia dell'autrice).

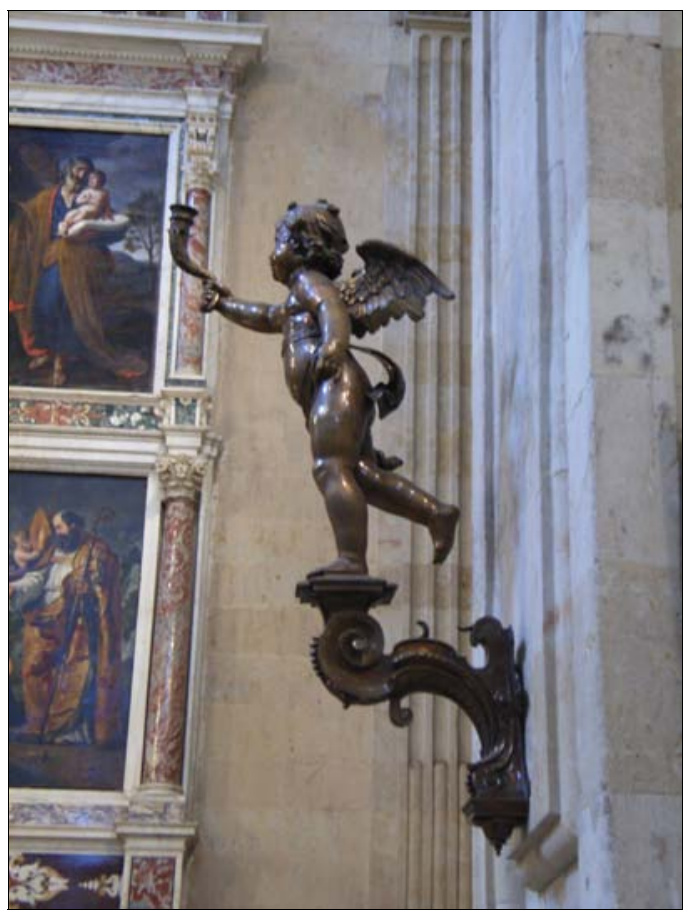

- Lám. 7. Putto reggitorciera. Salamanca. Chiesa de Las Agustinas Recoletas. Altare Maggiore. (Fotografia dell'autrice).

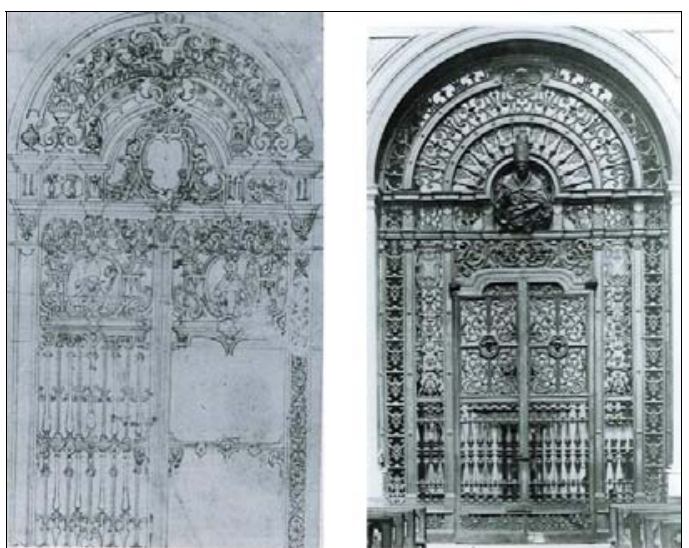

- Lám. 6. Disegno preparatorio per una cancellata. Londra. Courtauld Institute (Lascito Blunt). (da Civiltà del Seicento a Napoli, catalogo della mostra, vol. II); Cancello della Cappella del Tesoro di San Gennaro. Napoli. Duomo. (in Archivio Fotografico di Castel Sant'Elmo della Soprintendenza Speciale per il Polo Museale Napoletano).

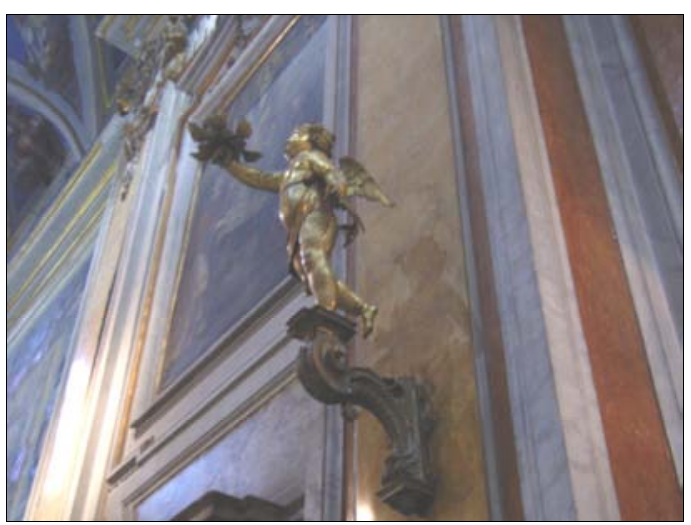

- Lám. 8. Putto reggitorciera. Napoli. Chiesa di Santa Maria la Nova. Altare Maggiore. (Fotografia dell'autrice). 


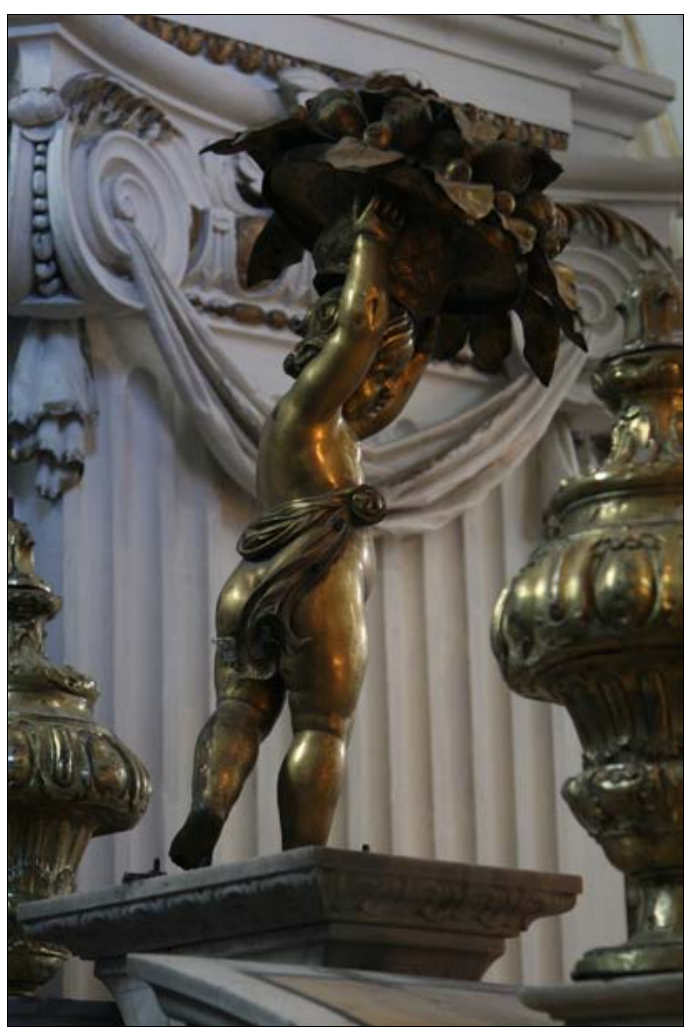

- Lám. 9. Putto reggi-canestro. Serra San Bruno(ViboValentia). Chiesa dell'Addolorata. (Fotografia di Raffaele Casciaro).

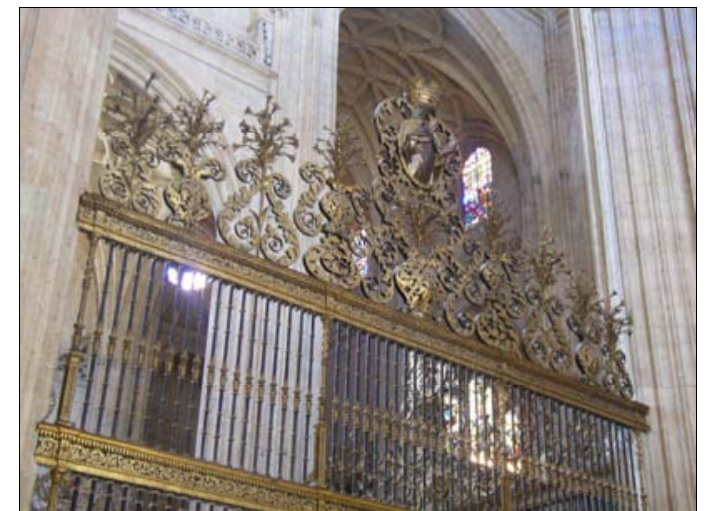

- Lám. 10. Cancellata. Segovia. Cattedrale. Capilla Mayor. (Fotografia dell'autrice).

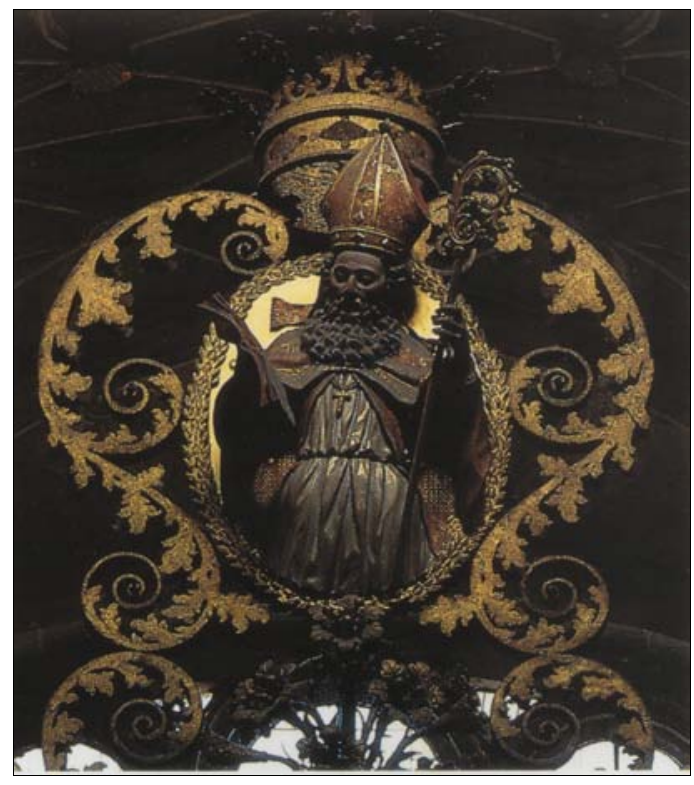

- Lám. 11. Cancellata. Particolare. Segovia. Cattedrale. Capilla Mayor. (Fotografia dell'autrice). 